\title{
POSSIBLE PROTO-PLANETARY NEBULAE
}

\author{
M. Parthasarathy \\ Indian Institute of Astrophysics
}

\begin{abstract}
IRAS data for high-galactic-latitude $F$ supergiants, a few luminous $F-G$ stars, and a few peculiar (forbidden) emission-line stars reveal they have dust shells with characteristics similar to those observed in planetary nebulae. The objects described here appear to have experienced severe mass loss in the recent past on the AGB. These stars are most likely post-AGB stars or evolving from the tip of AGB towards the lef $t$ in the $H-R$ diagram and may be described as possible proto-planetary nebulae. From the IRAS data we find ten additional possible proto-planetary nebulae. From the IRAS data of $\mathrm{HD} 56126,-53^{\circ} 5072$, HD 168625 and $-59^{\circ} 6723$ the luminosities, temperatures and masses of the dust shells are derived. HD 56126 (F5I), HD 168625 (B8Iae), and $-59^{\circ} 6723$ (B8Iae) appear to be in an evolutionary stage (post-AGB) similar to that of $\mathrm{HD} 161796$ and $\mathrm{HR} 4049$.
\end{abstract}

\section{INTRODUCTION}

The asymptotic giant branch (AGB) phase of evolution of intermediate and low mass stars is terminated by the ejection of the most of the hydrogen-rich outer envelope, resulting in a planetary nebula (Iben and Renzini 1983; Iben 1985). The superwind type of mass loss may lead to the formation of a planetary nebula and the termination of the AGB phase (Renzini, 1981). The Reimers mass-loss rate or low mass-loss rate will not be able to account for the formation of planetary nebulae. The formation of a planetary nebula may be preceded by a mass-loss rate of the order of $10^{-4}$ to $10^{-5}$ Mo per year (Zuckerman 1978). Renzini (1981) proposed that superwind type of mass loss $\left(10^{-4}\right.$ to $10^{-5}$ Me per year) can take place for low and intermediate mass stars at the tip of the AGB phase. Iben and Renzini (1983) showed that once the star reaches a critical luminosity most of the residual hydrogen-rich envelope is ejected on a time scale very short compared with the previous AGB life time. Sabbadin et al., (1984) showed that the radius-expansion velocity diagram for planetary nebulae is accounted better by the superwind type of phenomenon. Recently several evolved stars and OH/IR stars have been found to show mass loss rate of the order of $10^{-4}$ to $10^{-5}$ Mo per year (Knapp and Morris, 1985; Zuckerman and Dyck 1986a; Knapp 1987) which will become planetary nebulae within $10^{5}$ years. Pottasch's (1984) definition of proto-planetary nebulae includes objects at the tip of AGB and also objects in a transition stage from the tip of $A G B$ to the left in the HR diagram. 
From IRAS data we find that the high.galactic latitude $F$ supergiants, a few luminous $F-G$ stars, and a few peculiar (forbidden) emission-line stars to have dust shells with characteristics simflar to that observed in planetary nebulae. These objects appear to have experienced severe mass loss in the recent past on the AGB. These objects may be proto-planetary nebulae. In this paper we present a brief report on these objects. From the IRAS data ten additional possible proto-planetary nebulae are detected and their properties are reported.

\section{HIGH GALACTIC LATITUDE A AND F SUPERGIANTS}

The presence of $\mathrm{F}$-type supergiants at high galactic latitude was first noticed by Bidelman (1951). The evolutionary stage of these stars is not clear. HD 161796 and other stars in this group show small amplitude light and radial-velocity variations and also show switching of pulsation modes (Fernie 1986). The far infrared IRAS measurements of high galactic latitude $F$ supergiants $\mathrm{HD} 161796$ (F3Ib) and $\mathrm{HD} 187885$ (SAO 163075) (F2I) and related stars were found to show strong far infrared excess due to large amounts of circumstellar dust (Parthasarathy and Pottasch 1986). The masses and sizes of the dust envelopes around $\mathrm{HD}$ 161796 and $\mathrm{HD} 187885$ suggest that these stars suffered extensive mass loss in the recent past during the AGB stage of evolution. If the ratio of gas to dust mass is about 100 , as it is in the interstellar medium, the total shell masses are of the order of $0.3 \mathrm{Mo}$. This is very similar to that found in some planetary nebulae. Lamers et al. (1986) and Waelkens et al. (1987) found the high-galactic-latitude A supergiants HR 4049 and HD 213985 also to have far infrared (IRAS) excess due to dust shells. The evolutionary stage of these two stars also appears to be similar to that of $\mathrm{HD} 161796$ and $\mathrm{HD} 187885$.

Recently we found ten additional luminous F-G stars to show far infrared (IRAS) exesss due to dust shells around them (Pottasch and Parthasarathy 1988). Their far infrared flux distributions and colours are similar to that of $\mathrm{HD} 161796$ and planetary nebulae. The star HD 179821 (G5Ia) shows very large far infrared excess (12um: $31.4 \mathrm{jy}, 25 \mu \mathrm{m}$ : $647.7 \mathrm{jy} ; 60 \mu \mathrm{m}: 515.8 \mathrm{jy}$; and $100 \mu \mathrm{m}: 166.8 \mathrm{jy}) . \quad$ A low-dispersion spectrum of $\mathrm{HD} 179821$ obtained with the image tube spectrograph and 1 meter telescope at the Kavalur Observatory shows that its spectrum is very similar to that of a G5Ia star. The MK - M(v) calibration yields an absolute magnitude $M(v)=-8.0$. The luminosity of the dust shell is found to be $2.5 \times 10^{4}$ Lo, and the mass of the dust shell is estimated to be $2.6 \times 10^{-3}$ Mo. If the ratio of gas to dust mass is about 100 , as it is in the interstellar medium, the total shell mass is of the order of $0.26 \mathrm{Me}$. Zuckerman and Dyck (1986b) detected $C O \mathrm{~J}=1 \rightarrow 0$ emission from $\mathrm{HD} 179821$. It has the largest radial velocity with respect to local standard of rest. Its $\infty$ outflow velocity is also large $\left(34 \mathrm{~km} \mathrm{~s}^{-1}\right)$, which suggests that the star is of high luminosity. Zuckerman and Dyck (1986b) estimate the distance to be $6 \mathrm{kpc}$ and suggest that it is probably an unusual oxygen-rich supergiant located far from the galactic plane. The optical region spectra of $\mathrm{HD} 161796, \mathrm{HD} 187885$, and $\mathrm{HD} 179821$ clearly shows that they are high luminosity objects and the presence of detached cool dust shells suggests that they have experienced severe mass loss in the recent past. Detailed observational study of these stars may enable us to understand their evolutionary stage. 


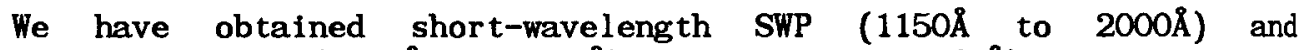

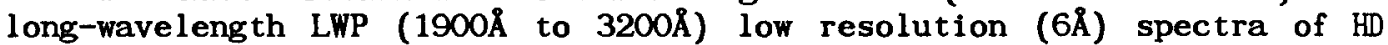
161796 and HD 188885 (only SWP spectrum) with the IUE satellite. The UV spectrum of $\mathrm{HD} 161796$ shows no excess UV flux attributable to a hot degenerate companion. The ultraviolet spectrum of $\mathrm{HD} 161796$ is consistent with it being a F3I star (Parthasarathy, Pottasch, Wamsteker 1988). The discontinuity at $1700 \AA$ due to SiI and the flux ratio $\log [f(\lambda)$ $(5500 \AA) / f(\lambda)(1900 \AA)]$, with the calibration of Bohm-Vitense (1982), yields a temperature of $6300 \mathrm{~K}$ for $\mathrm{HD}$ 161796, which is in good agreement with the value detrmined by Fernie and Garrison (1984). There is no evidence for significant metal deficiency. From an analysis of the UV spectrum we find that the metal abundance is normal: $[\mathrm{Fe} / \mathrm{H}]=0.0$. The $2000 \AA$ feature in the spectrum of $\mathrm{HD} 161796$ shows no evidence for circumstellar or interstellar reddening. In spite of large IR (IRAS) excess and large amount of dust around the star, there is no evidence for reddening from $1600 \AA$ to $3.5 \mu \mathrm{m}$. This result suggests that the grain size in the dust shell is relatively larger causing wavelength independent extinction.

The blue-visual region spectrum of $\mathrm{HD} 187885$ suggests that it is a F2I supergiant (Parthasarthy, Pottasch, Wamsteker 1988). However, the $1250 \AA$ to $1900 \AA$ low-resolution spectrum of $\mathrm{HD} 187885$ is pecullar because of the presence of a strong emission feature around $1580 \AA$ and the possibility of a broad absorption feature in the $1657 \AA$ spectral region. In late $A$ and early $F$ stars the flux in the wavelength region $\lambda<1700 \AA$ is very low. However, the UV spectrum of HD 187885 shows significant flux in the wavelength region $1400 \AA$ - $1700 \AA$. The strong and broad ( $1560 \AA$ to $1610 \AA$ ) emission at $1580 \AA$ may be due to SiI lines, since there is a crowding of SiI lines in this wavelength region. These lines can be in emission if the temperature at the surface drops less steeply than in normal $A$ and $F$ superglants or there is an outward increase of temperature. This also makes the discontinuity at $1700 \AA$ due to SiI less steep, which seems to explain the observed less steep discontinuity at $1700 \AA$. A broad absorption feature nearly $100 \AA$ wide centered around $1657 \AA$ appears to be present. This absorption feature may be due to $\mathrm{CI}$ or to quasimolecular absorption of H2 (Parthasarathy, Pottasch, Wamsteker 1988). High resolution UV spectrum of $\mathrm{HD} 187885$ may enable us to understand the cause for the spectral peculiarities.

\section{ADDITIONAL POSSIBLE PROTO-PLANETARY NEBULAE}

A list of ten additional possible proto-planetary nebulae is given in Table 1. All these ten sources show far infrared flux distributions and colours similar to that observed in planetary nebulae. For some of them the optical counterpart is very faint. The flux ratio $f(I R) / f(v i s)$ is very high, which suggests that the optical counterpart is obscured by the dust envelope. The dust temperature $T(d)$, the luminosity of the dust shell L(IR), and the mass M(d) of the dust shell around HD 56126, HD 168625 and $-59^{\circ} 6723$ are estimated and are given in Table 2 .

HD 56126 is a F5I supergiant. The IRAS data shows a flux maximum around $25 \mu \mathrm{m}$ (table 1). The dust temperature is found to be $140 \mathrm{~K}$. Van Genderen, Van Driel and Greidanus (1986) made VBLUW photometric observations and found it to show circumstellar reddening. The distance is estimated to be $5 \mathrm{kpc}$. The far infrared luminosity of the dust shell is found to be of the order of $1.4 \times 10^{4}$ Le. A low dispersion spectrum obtained with the image-tube spectrograph at Kavalur Observatory with $1-m$ 
Table 1:Additional Possible Proto-Planetary Nebulae

IRAS fluxes (Jansky)

\begin{tabular}{|c|c|c|c|c|c|c|c|c|c|c|c|c|}
\hline IRAS & $\begin{array}{l}\mathrm{R} \\
\mathrm{h} \\
07\end{array}$ & $\begin{array}{c}\text { A } \\
\mathrm{m} \\
02\end{array}$ & $\begin{array}{l}(1950) \\
\text { s } \\
45.3\end{array}$ & $\begin{array}{r}\mathrm{DF} \\
-79^{\circ}\end{array}$ & $34^{\prime}$ & 23 & V & $\mathrm{Sp}$ & 22.8 & $25 \mu \mathrm{m}$ & $60 \mu \mathrm{m}$ & $100 \mu \mathrm{m}$ \\
\hline $\begin{array}{l}\text { HD } 56126 \\
\text { SAO } 96709\end{array}$ & 07 & 13 & 25.4 & 10 & 05 & 08 & 8.2 & E5I & 24.6 & 116.7 & 49.8 & 18.2 \\
\hline RAFGL 4106 & 10 & 21 & 32.5 & -59 & 16 & 53 & & & 200.8 & 1754.6 & 851.7 & 179.6 \\
\hline $\begin{array}{l}-53^{\circ} 5072 \\
\text { SAO239853 }\end{array}$ & 12 & 17 & 33.3 & -53 & 38 & 49 & 9.3 & F2 & 1.0 & 20.7 & 7.4 & 2.4 \\
\hline $\begin{array}{l}-59^{\circ} 6723 \\
\text { SAO2 } 43756\end{array}$ & 16 & 20 & 40.5 & -59 & 56 & 38 & 9.8 & $\mathrm{~B} 8 \mathrm{Ia}$ & 0.4 & 11.1 & 12.1 & 4.7 \\
\hline IRAS & 16 & 34 & 17.1 & -38 & 14 & 18 & & & 16.3 & 199.8 & 290.1 & 138.2 \\
\hline IRAS & 16 & 59 & 26.7 & -46 & 56 & 14 & & & 45 & 297.3 & 130.5 & 33.5 \\
\hline RAFGL6815 & 17 & 15 & 04.6 & -32 & 24 & 15 & & & 58 & 321.4 & 268.3 & 81.4 \\
\hline $\begin{array}{l}\text { HD } 168625 \\
\text { Hen } 1681\end{array}$ & 18 & 18 & 25.5 & -16 & 23 & 56 & 8.4 & B8. Ia & 70. & 325.6 & 116.6 & \\
\hline IRAS & 20 & 02 & 48 & 39 & 10 & 03 & & & 41.8 & 210.8 & 142.6 & 45.9 \\
\hline
\end{tabular}

Table 2:Luminosities, Temperatures and Masses of the Dust Envelopes

\begin{tabular}{|c|c|c|c|c|c|}
\hline star & $d(k p c)$ & $F_{1 R}\left(10^{-12} \mathrm{Wm}^{-2}\right)$ & IIR $\quad(L \odot)$ & $\operatorname{Ta}(K)$ & Md $\left(M_{0}\right)$ \\
\hline HD 56126 & 5.2 & 16.5 & $1.4 \times 10^{4}$ & 140 & $3.8 \times 10^{-4}$ \\
\hline-535072 & & 2.6 & & & \\
\hline HD 168625 & 6.2 & 43.9 & $5.3 \times 10^{4}$ & 140 & $1.4 \times 10^{-3}$ \\
\hline-596723 & 5.6 & 1.8 & $1.8 \times 10^{3}$ & 100 & $1.8 \times 10^{-4}$ \\
\hline
\end{tabular}


telescope is very similar to that of a F4la supergiant. Spectra obtained in the red region show $\mathrm{H \alpha}$ in emission. The $\mathrm{H \alpha}$ emission strength and profile appears to be variable. Zuckerman. Dyck and Claussen (1986) find $\infty \mathrm{J}=1 \rightarrow 0$ emission (see also Likkel et al 1987). The IRAS data (Table 1) suggests that $\mathrm{HD} 56126$ is in an evolutionary stage similar to that of HD 161796.

HD 168625 is a B8Ia supergiant. The IRAS data shows a flux maximum at $25 \mathrm{um}$. It is also listed as an emission line object (Hen 1681). Near IR data also shows an infrared excess. The IRAS data yields a dust temperature of $140 \mathrm{~K}$. The distance is estimated to be about $3 \mathrm{kpc}$. The luminosity of the dust shell is found to be $1.2 \times 10^{4} \mathrm{Lo}$, and the mass of the dust shell is found to be of the order of $M(d)=3.3 \times 10^{-4} \mathrm{Mo}$.

$-59^{\circ} 6723$ (SAO 243756) is a luminous star in the southern $m i l k y$ way (Stephenson and Sanduleak, 1971). The MK spectral type is B8Iae. Klare and Neckel (1977) made UBV and polarization observations and $f$ ind $V=$ 9.79, $\mathrm{B}-\mathrm{V}=0.32, \mathrm{U}-\mathrm{B}=0.26$. The distance is estimated to be $5.6 \mathrm{kpc}$. The far infrared flux distribution and colours are similar to that observed in planetary nebulae. HD 168625 (B8Ia) and $-59^{\circ} 6723$ (B8Ia) appear to be similar to HR 4049 and HD 213985 (Waelkens et al. 1987).

Some of the objects described earlier in the text and some of the objects given in Table 1 were found to show $\infty \mathrm{J}=1 \rightarrow 0$ emission and also $1612 \mathrm{MHZ} \mathrm{OH}$ maser emission similar to that observed from evolved stars. The far-infrared characteristics of the circumstellar dust of the stars described here are very similar to those observed in planetary nebulae. These objects are not associated with any star forming regions. Some of the these objects are very far from the galactic plane. The most likely explanation for the presence of dust envelopes around these stars is that they experienced severe mass loss in the recent past on the AGB. They may be evolving from the tip of the AGB towards the lef $t$ in the HR diagram and may be described as possible proto-planetary nebulae.

As proto-planetary nebulae evolve into early stages of planetary nebulae, part of the dust shell will begin to be ionized. We find few peculiar emission line (forbidden) stars to show far infrared (IRAS) characteristics similar to that observed in planetary nebulae. From the IRAS data we find peculiar emission line stars HD 51585. Hen 401, Hen 591, Hen 1013, He2-138, M2-9, Hen 1336, Hen 1357, Hen 1428, Tc 1, and M1-26 to show dust shells with characteristics similar to that observed in young compact planetary nebulae (Parthasarathy and Pottasch 1988). The central stars in these objects are rapidly evolving towards hotter spectral types. He2-138, Tc 1 and M1-26 are clearly far enough advanced and may be called planetary nebulae. Recently quite a number of sources have been alleged to be objects in the transition between the tip of AGB and planetary nebula stage. There are approximately less than 200 possible proto-planetary nebulae in the IRAS point source catalogue. The duration of the transition stage is relatively short and therefore we expect to see significant differences in the characteristics of the objects occupying the transition region. The transition region may include objects like non-variable OH/IR stars (Le Bertre 1987; Kwok et al. 1987) and also objects like CRL 618, IRC +10420 , Vy 2-2, CRL 2688, and HD 161796, and on the other end of the transition there are young planetary nebulae like NGC 7027. NGC 6302, and IC 418. Detailed study of the objects in the transition region will enable us to understand their evolutionary stage and evolutionary sequence. 


\section{CONCLUSIONS}

The far infrared (IRAS) flux distributions and colours of the stars described here suggests that the dust envelopes around these stars have characteristics similar to that observed in planetary nebulae and evolved stars. The characterics of $\infty \mathrm{O}$ and $\mathrm{OH}$ emission from these objects also suggests that they are evolved stars. Objects of the type described here are not found in young clusters, associations, or in star forming regions. The presence of dust envelopes with characteristics similar to those observed in planetary nebulae suggests that they suffered severe mass loss in the recent past on the $A G B$. The CNO abundances and ${ }^{12} \mathrm{C} /{ }^{13} \mathrm{C}$ isotope ratio (from $\infty \mathrm{J}=1 \rightarrow 0$ and $2 \rightarrow 1$ data) of these stars may enable us to understand their evolutionary stage. The relation and evolutionary sequence between the stars described here and stars such as OH/IR 17.7-2.0 (Le Bertre 1987) and OH/IR 349.2-0.2, OH/IR 0.9+1.3 (Pottasch et al. 1987) is not clear, however, they all appear to be in a transition phase between the tip of the AGB and young planetary nebula stage and may be described as possible proto-planetary nebulae.

\section{REFERENCES}

Bidelman, W.P. 1951, Astrophys. J., 113, 304.

Bohm-Vitense, E. 1982, Astrophys. J., 225, 192.

Fernie, J.D. 1986, Astrophys. J., 306, 642.

Fernie, J.D. , Garrison, R.F. 1984, Astrophys. J., 285, 698.

Iben, I. 1985, Quart. J. Roy. Astron. Soc., 26, 1.

Iben, I., Renzini, A. 1983, Ann. Rev. Astron. Astrophys., 21, 271.

Klare, G.. Neckel, Th. 1977, Astron. Astrophys. Suppl ., 27, 215.

Knapp, G.R. 1987, in Late Stages of Stellar Evolution, ed. S. Kwok and S.R. Pottasch (Reidel:Dordrecht), p. 103.

Knapp, G.R., Morris, M. 1985, Astrophys. J., 292, 640.

Kwok, S. 1987, Astrophys. J., 321, 975.

Lamers, H.J.G.L.M., Waters, L.B.F.M. , Garmany, C.D., Perez, M.R., Waelkens, C. 1986, Astron. Astrophys., 154, L20.

Le Bertre, T. 1987, Astron. Astrophys., 180, 160.

Likkel, L., Omont, A., Morris, M., Forveille, T. 1987, Astron. Astrophys. 173. L11.

Parthasarathy, M. . Pot tasch, S.R. 1986, Astron. Astrophys., 154, L16.

Parthasarathy, M. . Pottasch, S.R., Wamsteker, W. 1988, Astron. Astrophys.. 203, 117.

Parthasarathy, M. , Pottasch, S.R. 1988, Astron. Astrophys., (submitted).

Pottasch, S.R. 1984, Planetary Nebulae, Astrophys. Space Science Library, Vol. 107.

Pottasch, S.R., Bignelli, C., Zijlstra, A. 1987, Astron. Astrophys., 177, L49.

Renzini, A. 1981, in Physical Processes in Red Giants ed. I. Iben, and A. Renzini (Dordrecht: Reidel), p. 165.

Sabbadin, F., Gratton, R.G., Blanchini, A., Ortolani, S. 1984, Astron. Astrophys. 136, 181. 
Stephenson, C.B., Sanduleak, N. 1971, Publ. Warner and Swasey Obs. Vol. 1. Van Genderen. A.M., Van Driel, W., Greidanus, H. 1986, Astron. Astrophys. 155. 72 .

Waelkens, C., Waters, L.B.F.M., Ccassatella, A., Le Bertre, T., Lamers, H.J.G.L.M. 1987, Astron. Astrophys. 181, L5.

Zuckerman, B. 1978, in Planetary Nebulae, ed. Y. Terzian (Reidel: Dordrecht), p. 305.

Zuckerman, B., Dyck, H.M. 1986a, Astrophys. J. (Letters), 304, 394.

Zuckerman, B., Dyck, H.M. 1986b, Astrophys. J. 311, 345.

Zuckerman, B., Dyck, H.M., Claussen, M.J. 1986, Astrophys. J. 304, 401. 